\title{
Automated ASPECTS in Acute Ischemic Stroke: A Comparative Analysis with CT Perfusion
}

\author{
(D) V.K. Sundaram, (D). Goldstein, (DD. Wheelwright, (D)A. Aggarwal, (D)P.S. Pawha, (D)A. Doshi, (D).T. Fifi, (D) R. De Leacy,
} (D). Mocco, (D). Puig, and (D). Nael

\begin{abstract}
BACKGROUND AND PURPOSE: Automated ASPECTS has the potential of reducing interobserver variability in the determination of early ischemic changes. We aimed to assess the performance of an automated ASPECTS software against the assessment of a neuroradiologist in a comparative analysis with concurrent CTP-based CBV ASPECTS.
\end{abstract}

MATERIALS AND METHODS: Patients with anterior circulation stroke who had baseline NCCT and CTP and underwent successful mechanical thrombectomy were included. NCCT-ASPECTS was assessed by 2 neuroradiologists, and discrepancies were resolved by consensus. CTP-CBV ASPECTS was assessed by a different neuroradiologist. Automated ASPECTS was provided by Brainomix software. ASPECTS was dichotomized (ASPECTS $\geq 6$ or $<6$ ) and was also based on the time from onset $(>6$ or $\leq 6$ hours).

RESULTS: A total of 58 patients were included. The interobserver agreement for NCCT ASPECTS was moderate $(\kappa=0.48)$ and marginally improved $(\kappa=0.64)$ for dichotomized data. Automated ASPECTS showed excellent agreement with consensus reads $(\kappa=0.84)$ and CTP-CBV ASPECTS $(\kappa=0.84)$. Intraclass correlation coefficients for ASPECTS across all 3 groups were $0.84(95 \% \mathrm{Cl}$, 0.76-0.90, raw scores) and 0.94 ( $95 \% \mathrm{Cl}, 0.91-0.96$, dichotomized scores). Automated scores were comparable with consensus reads and CTP-CBV ASPECTS in patients when grouped on the basis of time from symptom onset ( $>6$ or $\leq 6$ hours). There was significant $(P<.001)$ negative correlation with final infarction volume and the 3 ASPECTS groups $(r=-0.52$, consensus reads; -0.58, CTP-CBV; and -0.66 , automated).

CONCLUSIONS: ASPECTS derived from an automated software performs equally as well as consensus reads of expert neuroradiologists and concurrent CTP-CBV ASPECTS and can be used to standardize ASPECTS reporting and minimize interpretation variability.

ABBREVIATIONS: AIS = acute ischemic stroke; IQR = interquartile range

T he ASPECTS was initially developed in an effort to standardize assessment of the extent of early ischemic change on NCCT of the head in patients with acute ischemic stroke (AIS). ${ }^{1}$ The ASPECTS assessment has been increasingly incorporated in treatment decision-making and has been used in several randomized clinical trials for endovascular treatment decision-making. ${ }^{2,3}$ ASPECTS $\geq 6$ is now included in the latest version of the

Received July 4, 2019; accepted after revision September 18.

From the Department of Radiology (V.K.S., J.G., A.A., P.S.P., A.D., K.N.), Neuroimaging Advanced and Exploratory Lab, Department of Neurology (D.W., J.T.F., R.D.L.), and Department of Neurosurgery (J.T.F., R.D.L., J.M.), Icahn School of Medicine at Mount Sinai, New York, New York; and Department of Radiology (J.P.). University of Manitoba, Winnipeg, Manitoba, Canada.

Please address correspondence to Kambiz Nael, MD, Icahn School of Medicine at Mount Sinai, Department of Radiology, One Gustave L. Levy Place, New York, New York 10128; e-mail: kambiznael@gmail.com; @kambiznael

- Indicates open access to non-subscribers at www.ajnr.org

http://dx.doi.org/10.3174/ajnr.A6303
American Heart Association guidelines as an imaging eligibility criterion for endovascular treatment for patients presenting in the early treatment window ( $<6$ hours). ${ }^{4}$

The major drawback of the ASPECTS evaluation is its modest interobserver agreement and reproducibility. Early ischemic changes are often difficult to detect on NCCT, with low interobserver agreement for presence and extent. ${ }^{5-7}$

One solution to decrease variability in the detection of early ischemic changes is to use artificial intelligence and deep learning techniques to minimize the variability factor related to human interaction. ${ }^{8,9}$ Today automated software programs are commercially available to automatically calculate the ASPECTS using NCCT, with promising results in comparison with human interpretation. ${ }^{10-12}$

By providing hemodynamic and physiologic information, CTP delivers an improved delineation of early ischemia in comparison with NCCT. Applying ASPECTS to CTP-CBV maps has 
shown promising results in terms of the accuracy of detecting early ischemic changes, reduced variability, and prediction of functional outcome over NCCT ASPECTS., ${ }^{5,13-15}$

In this study, we aimed to evaluate the performance of automated ASPECTS obtained from a software-based analysis (Brainomix, Oxford, UK; www.brainomix.com) against the assessment of neuroradiologists in a comparative analysis with concurrent CT perfusion in a cohort of patients with acute ischemic stroke. This study was exploratory without a prespecified hypothesis.

\section{MATERIALS AND METHODS \\ Patient Selection}

This study was approved by the local institutional review board (Mount Sinai Health System, New York). We retrospectively reviewed consecutive patients with AIS who presented to our institution between January 2016 and July 2018 and met the following inclusion criteria: 1) time from symptom onset $<24$ hours, 2) anterior circulation ischemic stroke with large-vessel occlusion (intracranial carotid artery or MCA), 3) baseline NCCT, 4) technically adequate pretreatment CTP, and 5) successful recanalization defined by TICI (thrombolysis in cerebral infarction $) \geq 2$ b via mechanical thrombectomy. ${ }^{16}$

We recorded clinical data, including patients' age, sex, baseline NIHSS scores, time from stroke onset/last well known, location of large-vessel occlusion, time from CT to recanalization, data on receiving intravenous tissue plasminogen activator before mechanical thrombectomy, grade of recanalization using the TICI scale, and 90-day mRS when available. mRS scores of 0-2 were classified as indicating a good functional outcome.

\section{Image Acquisition}

CT image acquisition was performed using 2 CT scanners, including a LightSpeed VCT (GE Healthcare, Milwaukee, Wisconsin) and a Somatom Definition (Siemens, Erlangen, Germany). Helical NCCT $(120 \mathrm{kV}, 100-350$ auto-mA) was performed using a 5-mm section thickness from the foramen magnum through the vertex. CTP was performed with the following scan parameters: $100-\mathrm{mm}$ coverage in the z-axis, $80 \mathrm{kV}, 150 \mathrm{~mA}$, effective dose $=3.3 \mathrm{mSv}$, section thickness $=5 \mathrm{~mm}$, collimation $=64 \times 0.625 \mathrm{~mm}$. Total acquisition time was 60 seconds (30 consecutive spiral acquisitions, each 2 seconds). A total of $50 \mathrm{~mL}$ of iopamidol (Isovue-370; Bracco, Princeton, New Jersey) was injected intravenously followed by a $20-\mathrm{mL}$ saline flush at $5 \mathrm{~mL} / \mathrm{s}$.

\section{Image Analysis}

Two board-certified neuroradiologists independently reviewed all baseline NCCTs and assigned an ASPECTS using a 10-point scale. ${ }^{1}$ The site of arterial occlusion (right or left) was presented at the time of image interpretation. Discrepancies between 2 readers were resolved using a consensus read in a separate reading session.

In addition, an automated software-based analysis (Brainomix) was used to calculate automated ASPECTS (e-ASPECTS; https:// brainomix.com/e-aspects). Axial isotropic sequences from NCCT for each patient were uploaded to the software, and automated ASPECTS was calculated without human interaction. The neuroradiologists and automated ASPECTS were then exported to an Excel spreadsheet (Microsoft; Redmond, Washington) for analysis.

Subsequently, CTP data were processed using FDA-approved postprocessing software (Olea Sphere, Version 6.0; Olea Medical, La Ciotat, France). First, the arterial input function was detected automatically using a cluster-analysis algorithm. ${ }^{17}$ This arterial input function was subsequently used by the Bayesian probabilistic method ${ }^{18}$ to generate several perfusion parametric maps including CBV and CBF. To avoid recall bias, a boardcertified neuroradiologist (different from the one who determined ASPECTS on NCCT) reviewed CTP-CBV maps and assigned an ASPECTS for each patient.

The volume of final infarction was calculated by applying a volume of interest to the DWI hyperintense region using a voxelbased signal intensity method subsuming the entire region of DWI hyperintensity. When follow-up MR imaging was not available $(n=13)$, follow-up CT within 24-48 hours from thrombectomy was used for determination of final infarction volume. In these patients, infarct (defined as established hypodense regions) was manually delineated by a neuroradiologist with 10 years of experience.

\section{Statistical Analysis}

Clinical and demographic data were presented as mean (SD) or median (interquartile range) as appropriate. ASPECTS values were presented as median (interquartile range). Comparison of ASPECTS was performed using both the raw/original scores and dichotomized ASPECTS using $\geq 6$ and $<6$ as a cutoff. Interobserver agreement between 2 neuroradiologists was performed using a weighted $\kappa$ test with calculation of the $95 \%$ CI. Agreement and correlation among neuroradiologist consensus reads, CTP-CBV, and automated ASPECTS were performed using the intraclass correlation coefficient with 95\% CI. Comparative analysis between ASPECTS based on the time of symptom onset ( $<6$ or $>6$ hours) was also performed. Finally, clinical and imaging variables were compared between groups with good-versus-poor functional outcome (using 90-day mRS $>2$ as a cutoff), using a combination of $t$ tests and $\chi^{2}$ tests as appropriate.

\section{RESULTS}

\section{Demographic and Clinical Data}

A total of 58 patients met our inclusion criteria (27 men, 31 women; mean age, $69.9 \pm 12.5$ years). The mean of time from symptom onset was $8.4 \pm 5.3$ hours. The median and interquartile range (IQR) of the NIHSS were 15 and 10-21. A total of 42 patients had proximal middle cerebral artery occlusion, and 16 patients had intracranial internal carotid occlusion. Thirteen of 56 patients received IV-tPA before mechanical thrombectomy (this information was not available in 2 patients). The final mean infarct volume was $41 \pm 56 \mathrm{~mL}$. The median and IQR of 90 -day $\mathrm{mRS}$ available in 50 patients were 3 and $1-5$. The final recanalization (TICI) scores were $2 \mathrm{~b}(n=16), 2 \mathrm{c}(n=10)$, and $3(n=32)$.

\section{Human Interpretation}

The median for ASPECTS was 9 (IQR, 7-10) for reader 1 and 8 (IQR, 6-9) for reader 2. The interobserver agreement was moderate 
with $\kappa=0.48$ (95\% CI, 0.33-0.62). For the dichotomized ASPECTS (ASPECTS $\geq 6$ or $<6$ ), the interobserver agreement was improved with $\kappa=0.64$ (95\% CI, 0.32-0.95). For the final consensus read, the median for ASPECTS was 7 (IQR, 7-9). In the dichotomized consensus read, a total of 54 patients had ASPECTS $\geq 6$ and 4 patients had ASPECTS $<6$.

For CTP-CBV ASPECTS, the median was 8 (IQR, 7-9). A total of 50 patients had ASPECTS $<6$, while 8 had ASPECTS $\geq 6$. When ASPECTS values were evaluated between CTP and NCCT (human consensus reads), there was excellent agreement $(\kappa=$ 0.84 ; 95\% CI, 0.62-1.0) for the dichotomized scores and moderate agreement ( $\kappa=0.57 ; 95 \% \mathrm{CI}, 0.38-0.76)$ for the raw scores.

\section{Automated ASPECTS of NCCT}

The median was 9 (IQR, 8-10) for automated ASPECTS. The breakdown of dichotomized ASPECTS (ASPECTS $\geq 6$ /ASPECTS $<6)$ was $52 / 6$ for automated ASPECTS. There was excellent agreement $(\kappa=0.84$; 95\% CI, $0.62-1.0)$ between automated and consensus dichotomized ASPECTS. In only 2 patients, the software overestimated the extent of early ischemic changes by providing an automated ASPECTS $<6$, while the score was $>6$ by consensus read.

There was also excellent agreement $(\kappa=0.84$; 95\% CI, $0.62-$ 1.0) between automated and CTP-CBV dichotomized ASPECTS. In only 2 patients did the software underestimate the extent of early ischemic changes by providing an automated ASPECTS $>6$, while the CTP-CBV ASPECTS was $<6$.

Intraclass correlation coefficients were 0.84 (95\% CI, 0.76-0.90) for the original ASPECTS and 0.94 (95\% CI, 0.91-0.96) for the dichotomized ASPECTS across all 3 groups (consensus, CTP-CBV, and automated ASPECTS). Comparative scatterplots of ASPECTS across all 3 groups are shown in Fig 1.

Figure 2 demonstrates an example of ASPECTS and comparative analysis among humans, software, and CTP.

Comparative analysis between ASPECTS values based on the time of symptom onset ( $<6$ or $>6$ hours) did not show any significant difference for neuroradiologists, CTP-CBV, or automated ASPECTS (Table 1).

In 50 patients who had a 90 -day mRS, 22 patients (44\%) had good functional outcome using 90-day mRS $\leq 2$, while 28 patients $(56 \%)$ had poor $(m R S>2$ ) functional outcome. Patients with poor functional

FIG 1. Comparative illustration of the distribution of ASPECTS from consensus reads of 2 neuroradiologists, software-based automated ASPECTS, and CTP-CBV for all patients in our study population (each marker represents a patient's score). Automated ASPECTS showed excellent agreement $(\kappa=0.84 ; 95 \% \mathrm{Cl}, 0.62-1.0)$ with both consensus and CTP-CBV ASPECTS. The intraclass correlation coefficient was $0.84(95 \% \mathrm{Cl}, 0.76-0.90)$ across all 3 groups.

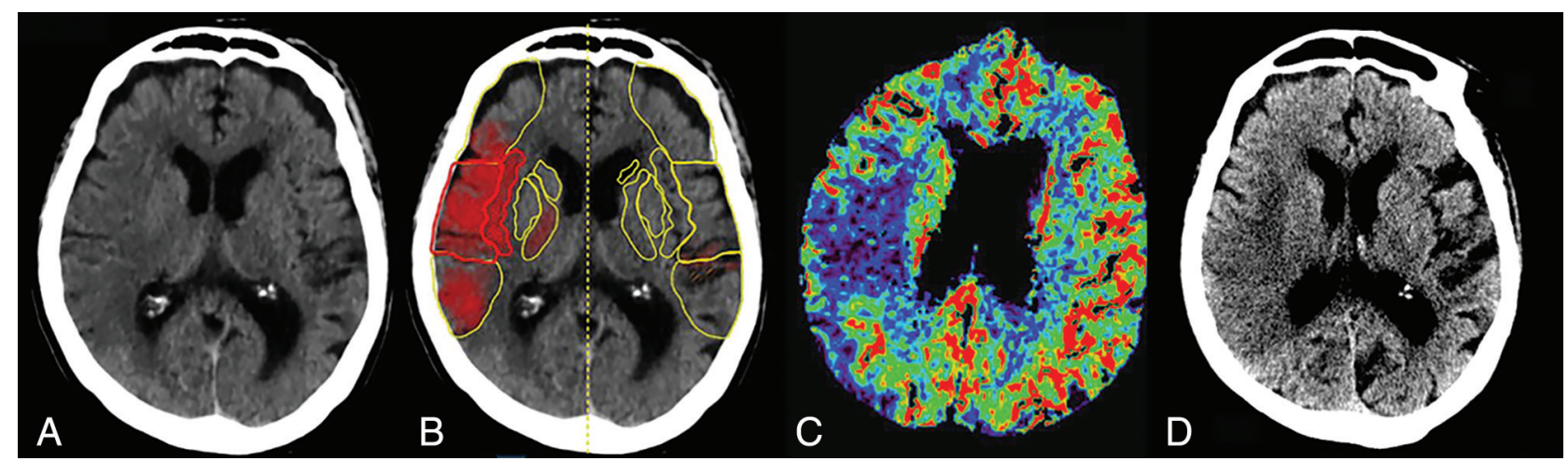

FIG 2. An 82-year-old woman who presented with right MCA M1 occlusion and an NIHSS score of 18. She underwent successful mechanical thrombectomy (TICl 3) with a CT-to-recanalization time of 50 minutes. Axial NCCT (A), automated ASPECTS (B), axial CTP-CBV (C), and axial NCCT 48 hours after endovascular treatment $(D)$ are shown. For the 2 human readers, one scored 6 and the other, 7 (consensus ASPECTS, 6 ). $B$, Automated software assigned an ASPECTS of 6. CTP-CBV ASPECTS was 7. There is good topographic correlation with the final infarction volume. 
outcome had significantly higher NIHSS $(P=.001)$, older age $(P=.02)$, and higher infarction volume $(P=.03)$. Of note, none of the ASPECTS values (consensus read, CTP-CBV, or eASPECTS) were discriminatory between patients with goodversus-poor functional outcome. Clinical and imaging variables compared in groups with good-versus-poor functional outcome are summarized in Table 2.

All 3 ASPECTS groups had significant $(P<.001)$ negative correlation with final infarction volume, with a correlation coefficient $(r)$ of -0.52 for the consensus reads, -0.58 for CTP-CBV, and -0.66 for automated ASPECTS. Figure 3 shows correlation scatterplots of ASPECTS and final infarction volumes.

\section{DISCUSSION}

ASPECTS has become increasingly integrated into the decisionmaking process for intervention in patients with AIS. According to current guidelines, absence of a large infarction core via NCCT defined by ASPECTS $\geq 6$ is sufficient for treatment decisionmaking in patients with anterior circulation large-vessel occlusion within the first 6 hours from the symptom onset. ${ }^{4}$ For the same population of patients if presenting between 6 and 24 hours from symptom onset, there is a lack of data supporting the use of

Table 1: Comparative analysis among ASPECTS scores categorized on the basis of time of symptom onset ${ }^{a}$

\begin{tabular}{lccc}
\hline & $\begin{array}{c}\text { Onset Symptoms } \\
\leq 6 \text { hrs (24) }\end{array}$ & $\begin{array}{c}\text { Onset Symptoms } \\
>6 \text { hrs (34) }\end{array}$ & $P$ Value \\
\hline $\begin{array}{l}\text { Consensus ASPECTS } \\
\quad(2 \text { neuroradiologists) }\end{array}$ & $8(6-9)$ & $7(7-9)$ & .46 \\
Automated ASPECTS & $9(7-10)$ & & \\
CTP-CBV ASPECTS & $8(6-9)$ & $9(8-9)$ & .64 \\
\hline
\end{tabular}

${ }^{a}$ Data are presented in median (IQR).
NCCT ASPECTS alone for treatment selection, ${ }^{19}$ and additional imaging including CTP or MR imaging is recommended. ${ }^{4}$

Our results reaffirmed the concern of interobserver variability for ASPECTS assessment by showing only fair interobserver agreement $(\kappa=0.48)$, even for experienced neuroradiologists. This has been attributed to factors such as physician training and experience, time pressure, and personal bias of expected findings (for example, from the ordering or treatment teams), among other factors that have been noted as potential reasons for the variability of ASPECTS. ${ }^{11,20,21}$ Similar to other investigators, using dichotomized ASPECTS ( $\geq 6$ or $<6$ ), we showed marginal improvement in interobserver agreement $(\kappa=0.64) .{ }^{13,22}$ However, there remains substantial variability in ASPECTS assessment, which can introduce uncertainty for all physicians involved in the care of patients with stroke and affect the clinical management and implementation of a guidelinebased approach for stroke treatment. ${ }^{4}$

With the introduction of software packages trained on deep learning algorithms, attempts have been made to use automated ASPECTS as a way to address variability associated with human interpretation with some success. ${ }^{10-12,23-25}$ In this study, we showed that automated ASPECTS has a similar diagnostic performance to consensus reading of experienced neuroradiologists with excellent agreement $(\kappa=$ 0.84 ). In a recent study by Maegerlein et $\mathrm{al}^{11}$ using a different software package, similar results were shown with substantial agreement $(\kappa=0.9)$ between automated and consensus reads. Most important, we showed that automated ASPECTS performed equally well compared with consensus reads

Table 2: Clinical and imaging data in patients with good-versus-poor functional outcome using $\mathbf{m R S}>\mathbf{2}$ as a cutoff

\begin{tabular}{lcccc}
\hline \multicolumn{1}{c}{ Variable } & Overall $(\boldsymbol{n}=\mathbf{5 0})$ & Good Outcome $(\boldsymbol{n}=\mathbf{2 2})$ & Poor Outcome $(\boldsymbol{n}=\mathbf{2 8})$ & $\boldsymbol{P}$ Value \\
\hline Age (mean) (SD) (yr) & $70.0(13.0)$ & $65.1(12.8)$ & $73.8(12.1)$ & $10: 18$ \\
Sex (M/F) & $23: 27$ & $13: 9$ & .02 \\
Baseline NIHSS (median) (IQR) & $15(11-21)$ & $11(8-14)$ & $20(14-22)$ & .10 \\
Time from symptom onset (mean) (SD) (hr) & $8.4(5.6)$ & $8.8(6.7)$ & .001 \\
Consensus ASPECTS $\geq 6$ (No.) (\%) & 43 & $21(95 \%)$ & $22(78 \%)$ & .62 \\
Automated ASPECTS $\geq 6$ (No.) (\%) & 45 & $21(95 \%)$ & $24(85 \%)$ & .10 \\
CTP-CBV ASPECTS $\geq 6$ (No.) (\%) & 43 & $21(95 \%)$ & .26 \\
Final infarction volume (mean) (SD) (mL) & 49 & $24.4(29.0)$ & $57.5(64.7)$ & .10 \\
\hline
\end{tabular}
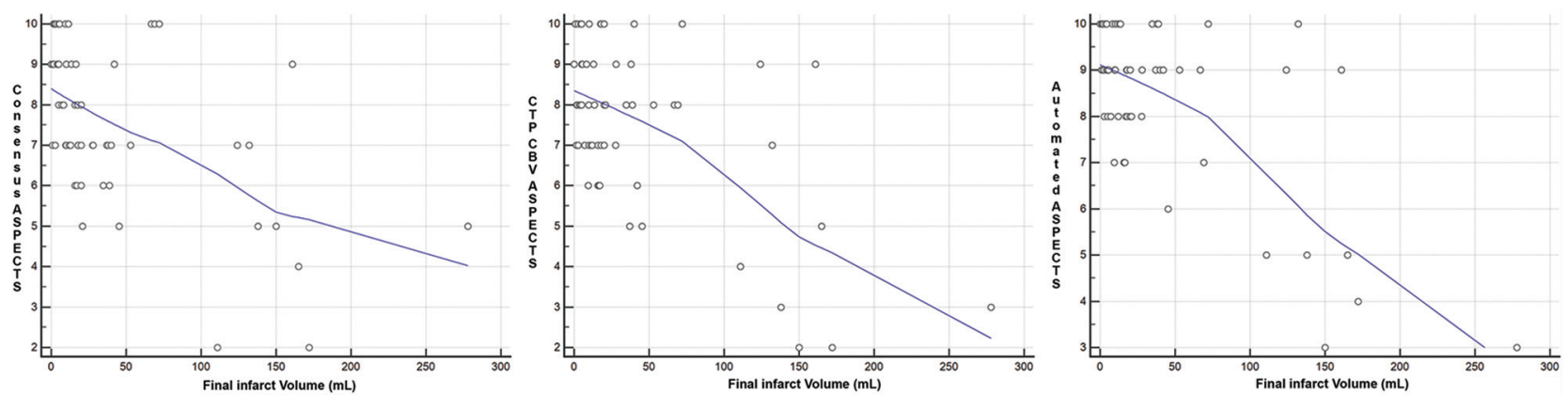

FIG 3. Scatterplots for correlation between ASPECTS and final infarction volume for all 3 ASPECTS groups showing significant $(P<.001)$ negative correlation ( $r=-0.52$ for the consensus reads, -0.58 for CTP-CBV, and -0.66 for automated ASPECTS). 
regardless of the time from onset ( $\geq 6$ or $<6$ hours). Assuming that consensus ASPECTS of 2 neuroradiologists can be used as an "operation criterion standard" in an acute setting, it is plausible to use automated ASPECTS to standardize NCCT interpretation in the acute setting and avoid variability associated with individual human interpretation, ensuring that all patients receive equivalent care and are triaged with appropriate treatment options.

Our second finding is that the described automated ASPECTS provides similar diagnostic performance to concurrently performed CTP-CBV ASPECTS. CTP provides more sensitive assessment of early ischemic changes in AIS because it contains physiologic and hemodynamic information about the ischemia. CTP ASPECTS has been used successfully in the triage of patients with AIS with the potential added value of improving reliability and reducing variability in the assessment of early ischemic changes compared with NCCT ASPECTS, in particular in earlier treatment windows. ${ }^{15,26-29}$ In a study of 227 patients, Naylor et $\mathrm{al}^{15}$ showed improvement in the reliability of early ischemic changes using CTP ASPECTS over NCCT ASPECTS.

In our study, having similar diagnostic performance and excellent agreement $(\kappa=0.84)$ between automated software and CTP is reassuring and strengthens the validity of the automated ASPECTS and the argument that it can be used in clinical practice with confidence. In our study using a cutoff for time from symptom onset at 6 hours that has been used in the current guidelines, we did not find any significant discrepancy among our ASPECTS groups.

Finally, we showed that none of our ASPECTS groups (human, automated, or CTP) were predictive of functional outcome as it was measured by 90 -day mRS. Although there are several reports supporting a favorable association between higher ASPECTS and good functional outcome, ${ }^{1,30,31}$ others have debated the ability of ASPECTS to predict outcome. ${ }^{20,32}$ There are also some reports that showed the predictive ability of CTP ASPECTS for determination of functional outcome. $^{27,33}$ In a recent study by Pfaff et al, ${ }^{24}$ automated ASPECTS was shown to be predictive of functional outcome. One reason for the lack of association between ASPECTS and functional outcome in our study could be an insufficient number of patients with ASPECTS $<6$ (only 6 patients using automated ASPECTS). In fact, $21 / 22$ patients who had good functional outcome had a baseline automated ASPECTS score of $\geq 6$.

Although ASPECTS values were not predictive of functional outcome in our study, they were predictive of final infarction volume concordant with prior reports. ${ }^{34}$ Using automated ASPECTS, a recent study by Demeestere et $\mathrm{al}^{34}$ showed findings similar to ours by demonstrating a lack of association with functional outcome but significant correlation in the determination of final infarction volume. We found 3 variables, including age, NIHSS, and final infarction volume, as significant contributors to predicting functional outcome. Most interesting, 2 of these (age and NIHSS) have been used in a prior established predictive score (Houston Intra-Arterial Therapy 2 score, which combines age, glucose level, NIHSS, and ASPECTS) for improved prediction of functional outcome in patients with AIS. ${ }^{35}$
There are several limitations to our study. Retrospective design can introduce unknown bias. There was selection bias because only patients with AIS were included. The sample size was relatively small, and further validation studies with larger sample sizes are required to validate the practical application of our automated software as a stand-alone tool in the triage of patients with AIS. Similarly, we had only a small group of patients with low ASPECTS, and this limits assessment of the association with functional outcome. Infarct extension and increased volume are possible between CT and the follow-up imaging after endovascular thrombectomy, which was used for final infarction determination. We tried to minimize this confounding factor by including patients with successful recanalization. Another limitation is that the final imaging study used for determination of final infarction volume was CT in a subset of patients (22\%), which is less than ideal in comparison with MR imaging and can possibly introduce inconsistencies into our analysis. Finally, the CTP-CBV ASPECTS values were assessed by only 1 observer.

\section{CONCLUSIONS}

We showed that automated ASPECTS provided by the described software performs equally well compared with a consensus read of expert neuroradiologists and concurrent CTP-CBV ASPECTS in patients presenting with acute ischemic stroke. If its potential is realized, automated ASPECTS can be used as a stand-alone tool for triage and treatment decision-making in patients with acute ischemic stroke.

Disclosures: J. Mocco—RELATED: Board Membership: Neurotechnology Investors; Consultancy: Imperative Care, Cerebrotech inc., Viseon, EndoStream Medical, Rebound Therapeutics, Vastrax; UNRELATED: Stock/Stock Options: Neuro-technology Investors, comet inc., Neurvana inc, Cardinal Consulting, Viseon, Echovate, Serenity, RIST, Spinaker, inc., Blockade Medical. Kambiz Nael—RELATED: Board Membership: Olea Medical, Comments: Medical Advisory Board.

\section{REFERENCES}

1. Barber PA, Demchuk AM, Zhang J, et al. Validity and reliability of a quantitative computed tomography score in predicting outcome of hyperacute stroke before thrombolytic therapy: ASPECTS Study Group-Alberta Stroke Programme Early CT Score. Lancet 2000;355:1670-74 CrossRef Medline

2. Goyal M, Demchuk AM, Menon BK, et al. Randomized assessment of rapid endovascular treatment of ischemic stroke. $N$ Engl J Med 2015;372:1019-30 CrossRef Medline

3. Saver JL, Goyal M, Bonafe A, et al. Stent-retriever thrombectomy after intravenous t-PA vs. t-PA alone in stroke. $N$ Engl J Med 2015;372:2285-95 CrossRef Medline

4. Powers WJ, Rabinstein AA, Ackerson T, et al. 2018 Guidelines for the Early Management of Patients With Acute Ischemic Stroke: A Guideline for Healthcare Professionals From the American Heart Association/American Stroke Association. Stroke 2018;49:e46-e110 CrossRef Medline

5. Finlayson O, John V, Yeung R, et al. Interobserver agreement of ASPECT score distribution for noncontrast CT, CT angiography, and CT perfusion in acute stroke. Stroke 2013;44:234-36 CrossRef Medline

6. Mak HK, Yau KK, Khong PL, et al. Hypodensity of $>1 / 3$ middle cerebral artery territory versus Alberta Stroke Programme Early CT Score (ASPECTS): comparison of two methods of quantitative evaluation of early CT changes in hyperacute ischemic stroke in the community setting. Stroke 2003;34:1194-96 CrossRef Medline 
7. Wardlaw JM, Dorman PJ, Lewis SC, et al. Can stroke physicians and neuroradiologists identify signs of early cerebral infarction on CT? J Neurol Neurosurg Psychiatry 1999;67:651-53 CrossRef Medline

8. Bentley P, Ganesalingam J, Carlton Jones AL, et al. Prediction of stroke thrombolysis outcome using CT brain machine learning. Neuroimage Clin 2014;4:635-40 CrossRef Medline

9. Tang FH, Ng DK, Chow DH. An image feature approach for computer-aided detection of ischemic stroke. Comput Biol Med 2011; 41:529-36 CrossRef Medline

10. Herweh C, Ringleb PA, Rauch G, et al. Performance of e-ASPECTS software in comparison to that of stroke physicians on assessing CT scans of acute ischemic stroke patients. Int J Stroke: 2016;11: 438-45 CrossRef Medline

11. Maegerlein C, Fischer J, Monch S, et al. Automated calculation of the Alberta Stroke Program Early CT Score: feasibility and reliability. Radiology 2019;291:141-48 CrossRef Medline

12. Nagel S, Sinha D, Day D, et al. e-ASPECTS software is non-inferior to neuroradiologists in applying the ASPECT score to computed tomography scans of acute ischemic stroke patients. Int J Stroke 2017;12:615-22 CrossRef Medline

13. Aviv RI, Mandelcorn J, Chakraborty S, et al. Alberta Stroke Program Early CT Scoring of CT perfusion in early stroke visualization and assessment. AJNR Am J Neuroradiol 2007;28:1975-80 CrossRef Medline

14. Parsons MW, Pepper EM, Chan V, et al. Perfusion computed tomography: prediction of final infarct extent and stroke outcome. Ann Neurol 2005;58:672-79 CrossRef Medline

15. Naylor J, Churilov L, Chen Z, et al. Reliability, reproducibility and prognostic accuracy of the Alberta Stroke Program Early CT Score on CT perfusion and non-contrast CT in hyperacute stroke. Cerebrovasc Dis 2017;44:195-202 CrossRef Medline

16. Tomsick T, Broderick J, Carrozella J, et al. Revascularization results in the Interventional Management of Stroke II trial. AJNR Am J Neuroradiol 2008;29:582-87 CrossRef Medline

17. Mouridsen K, Christensen S, Gyldensted L, et al. Automatic selection of arterial input function using cluster analysis. Magn Reson Med 2006;55:524-31 CrossRef Medline

18. Boutelier T, Kudo K, Pautot F, et al. Bayesian hemodynamic parameter estimation by bolus tracking perfusion weighted imaging. IEEE Trans Med Imaging 2012;31:1381-95 CrossRef Medline

19. Saver JL, Goyal M, van der Lugt A, et al. Time to treatment with endovascular thrombectomy and outcomes from ischemic stroke: a meta-analysis. JAMA 2016;316:1279-88 CrossRef Medline

20. McTaggart RA, Jovin TG, Lansberg MG, et al. Alberta stroke program early computed tomographic scoring performance in a series of patients undergoing computed tomography and MRI: reader agreement, modality agreement, and outcome prediction. Stroke 2015;46:407-12 CrossRef Medline

21. Kobkitsuksakul C, Tritanon O, Suraratdecha V. Interobserver agreement between senior radiology resident, neuroradiology fellow, and experienced neuroradiologist in the rating of Alberta Stroke Program Early Computed Tomography Score (ASPECTS). Diagn Interv Radiol 2018;24:104-07 CrossRef Medline

22. Gupta AC, Schaefer PW, Chaudhry ZA, et al. Interobserver reliability of baseline noncontrast CT Alberta Stroke Program Early CT
Score for intra-arterial stroke treatment selection. AJNR Am J Neuroradiol 2012;33:1046-49 CrossRef Medline

23. Kuang H, Najm M, Chakraborty D, et al. Automated ASPECTS on noncontrast CT scans in patients with acute ischemic stroke using machine learning. AJNR Am J Neuroradiol 2019;40:33-38 CrossRef Medline

24. Pfaff J, Herweh C, Schieber S, et al. e-ASPECTS correlates with and is predictive of outcome after mechanical thrombectomy. AJNR Am J Neuroradiol 2017;38:1594-99 CrossRef Medline

25. Guberina N, Dietrich U, Radbruch A, et al. Detection of early infarction signs with machine learning-based diagnosis by means of the Alberta Stroke Program Early CT Score (ASPECTS) in the clinical routine. Neuroradiology 2018;60:889-901 CrossRef Medline

26. Padroni M, Boned S, Ribo M, et al. CBV_ASPECTS improvement over CT_ASPECTS on determining irreversible ischemic lesion decreases over time. Intervent Neurol 2016;5:140-147 CrossRef Medline

27. Padroni M, Bernardoni A, Tamborino C, et al. Cerebral blood volume ASPECTS is the best predictor of clinical outcome in acute ischemic stroke: a retrospective, combined semi-quantitative and quantitative assessment. PLoS One 2016;11:e0147910 CrossRef Medline

28. Liu ZW, Jiang Y, Wang R, et al. Combined application of multi-parameter semiquantitative Alberta Stroke Program Early CT Score to assess infarction severity in acute ischemic stroke [In Chinese; Abstract available in Chinese from the publisher]. Zhonghua Yi Xue Za Zhi 2018;98:1697-1702 CrossRef Medline

29. Psychogios MN, Knauth M, Bshara R, et al. Computed tomography perfusion-based selection of endovascularly treated acute ischaemic stroke patients: are there lessons to be learned from the preevidence era? Neuroradiol J 2017;30:138-43 CrossRef Medline

30. Olive-Gadea M, Martins N, Boned S, et al. Baseline ASPECTS and eASPECTS correlation with infarct volume and functional outcome in patients undergoing mechanical thrombectomy. J Neuroimaging 2019;29:198-202 CrossRef

31. Dzialowski I, Hill MD, Coutts SB, et al. Extent of early ischemic changes on computed tomography (CT) before thrombolysis: prognostic value of the Alberta Stroke Program Early CT Score in ECASS II. Stroke 2006;37:973-78 CrossRef Medline

32. Weir NU, Pexman JH, Hill MD, et al. How well does ASPECTS predict the outcome of acute stroke treated with IV tPA? Neurology 2006;67:516-18 CrossRef Medline

33. Lee JY, Kim SH, Lee MS, et al. Prediction of clinical outcome with baseline and 24-hour perfusion CT in acute middle cerebral artery territory ischemic stroke treated with intravenous recanalization therapy. Neuroradiology 2008;50:391-96 CrossRef Medline

34. Demeestere J, Scheldeman L, Cornelissen SA, et al. Alberta Stroke Program Early CT Score versus computed tomographic perfusion to predict functional outcome after successful reperfusion in acute ischemic stroke. Stroke 2018;49:2361-67 CrossRef Medline

35. Sarraj A, Albright $\mathrm{K}$, Barreto $\mathrm{AD}$, et al. Optimizing prediction scores for poor outcome after intra-arterial therapy in anterior circulation acute ischemic stroke. Stroke 2013;44:3324-30 CrossRef Medline 